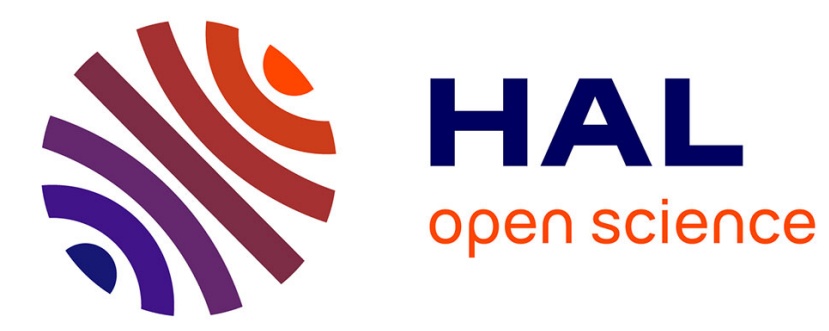

\title{
New spectral image compression method based on an optimal phase coding and the RMS duration principle
}

\author{
Ayman Alfalou, Marwa El Bouz, Ali Mansour, Gilels Keryer
}

\section{To cite this version:}

Ayman Alfalou, Marwa El Bouz, Ali Mansour, Gilels Keryer. New spectral image compression method based on an optimal phase coding and the RMS duration principle. Journal of Optics, 2010, 12, 115403 (12 pp). hal-00579175

\section{HAL Id: hal-00579175 \\ https://hal.science/hal-00579175}

Submitted on 23 Mar 2011

HAL is a multi-disciplinary open access archive for the deposit and dissemination of scientific research documents, whether they are published or not. The documents may come from teaching and research institutions in France or abroad, or from public or private research centers.
L'archive ouverte pluridisciplinaire HAL, est destinée au dépôt et à la diffusion de documents scientifiques de niveau recherche, publiés ou non, émanant des établissements d'enseignement et de recherche français ou étrangers, des laboratoires publics ou privés. 


\title{
New spectral image compression method based on an optimal phase coding and the RMS duration principle
}

\author{
A. Alfalou, ${ }^{1}$ M. Elbouz, ${ }^{1}$ A. Mansour ${ }^{2}$, G. Keryer $^{1}$ \\ 1 Département optoélectronique, Laboratory L@BISEN, ISEN-BREST, 20 rue \\ cuirassé Bretagne, CS 42807, 29228 Brest Cedex 2, France. \\ 2 Department of Electrical and Computer Engineering, Curtin University of \\ Technology, GPO Box U1987, Perth, WA, 6845, Australia \\ Email : ayman.al-falou@isen.fr
}

\begin{abstract}
By only using phase information, this manuscript describes a new spectral lossy compression method which can: Reduce required memories; adaptively retrieve original images by only using spectral phase information; and be easily employed in major encryption techniques. To increase the compression ratio of the proposed method, an optimal phase coding based on "a fading grid" is performed. In fact, a variable number of quantization bits has been used to quantize phase information depending on the importance of the spectral phases. The phase information could be classified according to the concept of "RMS Duration". Many simulations have been carried out. Our experimental results corroborate the performance of the new proposed method. (42.30.Kq, 42.30.Va, 42.40.My)
\end{abstract}

Keywords: Fouier transform, Fourier plane, filters, image processing, images compression, segmentation, RMS-Duration.

\section{Introduction}

In the last decade, several optical compression and encryption methods have been developed. These methods can be used in the video and multimedia transmission [1]. In fact, optical methods are the most promising methods for parallelism, however they rely a lot on the developed technology of optoelectronic devices [1]. Furthermore, in a recent work [2], we have proposed and validated a spectral compression and encryption method based on a specific manipulation of the different target images spectra i.e. fusion without overlapping. Hereinafter, we propose a new spectral compression method which can be integrated in spectral filtering [3], form recognition [4-8], encryption [9-13], etc.

In this paper, the compression steps can be achieved at the optical level, i.e. at the origin of light images, and they are optimised in such way that they don't compromise the performance of the reconstructed image at the output plane of our system. In many applications such as face recognition, fingerprint identification and security check applications, CCD cameras or sensors are employed far away from data processing centers. In most cases, recorded images should be wirelessly transmitted to processing centers. For that, it is necessary to reduce the needed information to be stored or transmitted i.e. compression. Many recent algorithms have been proposed to deal with these applications. Recently, the authors of ref [14] have shown that classical compression methods, as JPEG, are not suitable for optical information processing. 
To overcome the previous constraints, our spectral compression method is mainly based on two important concepts. The first concept is based on the phasis use in Fourier domain whom their importance is clearly highlighted in [15]. In fact, Oppenheim et al [15] proved that the phasis of a spectrum can be used to retrieve the original image. This reconstruction is subject to few constraints emphasized in their paper. The second concept use iterative image reconstruction techniques [16]. In their manuscript [16], Quatieri et al proposed an iterative algorithm to reconstruct any image from the phasis of its spectrum. For multimedia and video applications, the number of needed iterations can be reduced by introducing estimated amplitude of a reference image.

We begin this paper by analyzing this kind of techniques in order to adapt it to our spectral compression method. In the following, only phase reconstruction methods are taken into consideration for the main following reasons:

- $\quad$ Phase information plays a major role in the image reconstruction procedure.

- Thanks to the phase limitation in $[-\pi,+\pi]$, we can efficiently reduce the number of bits required to quantize, record and process phase information [17].

- Recently, different researchers [18-20] have independently proposed compression methods which consider a variable bit number.

- $\quad$ The authors of [21] proved that an image could be reconstructed by only using "partial phase information localized in the Fourier domain".

For all the above mentioned reasons, our method allocates high bit numbers for the most important phase information. The selection of the most important phase information can be done with the concept of "RMS Duration" developed by Papoulis in 1962 [22]. Using this strategy, one could drastically reduce both the number of bits and the overall size of information to be recorded and/or transmitted.

\section{Analysis of Image reconstruction technique based only on phase information}

Since the eighties, many researchers like Oppenheim et al [15-16] have emphasized the importance of phase information in any image reconstruction algorithm. To illustrate this fact, Figure (1) shows the outcome of an inverse Fourier transform applied to the phase information (i.e. the spectral phase of the reconstructed image). As shown in Figure (1), a basic contour image can be easily obtained from the phase information. To improve the quality of the recontracted image, an iterative procedure has been put in place [15-16]. By satisfying some constraints, it was proved in [15-16] that a very good quality reconstructed image can be achieved by only manipulating the phase information [23].

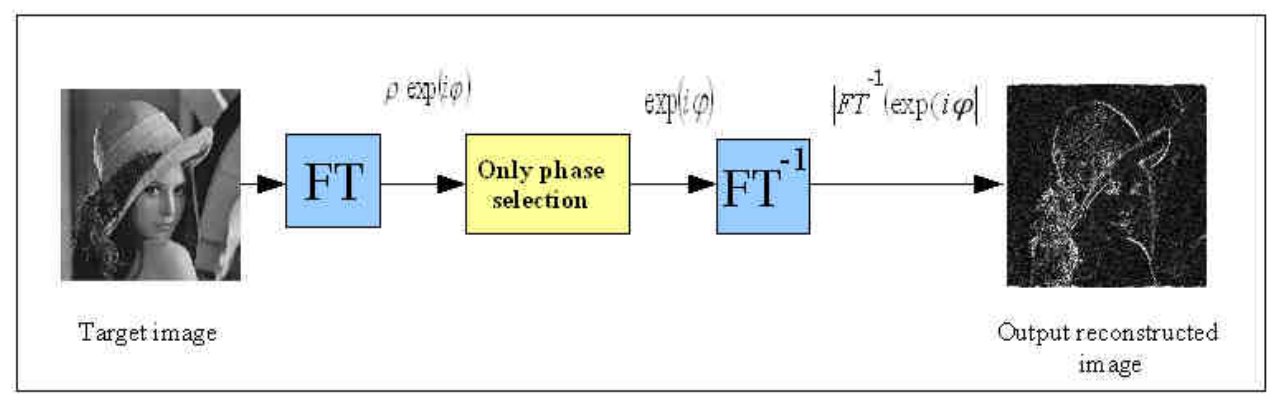

Figure 1. Reconstructed image. 


\subsection{Major steps of the Algorithm}

The new proposed algorithm (i.e. to storage information to be transmitted) has the advantage to be easy implemented, it doesn't require complex and exhausting computational efforts and it has good performance. The algorithm can be summarized by the following two parts, as shown in Figure (2):

1. Transmitter (Figure 2-a) is used to record the only spectrum phase needed to reconstruct an image. The first step (Figure 2-a) consists in adding zeros padding to the target image $(N \times N$ pixels). After that, we obtain an input image with $(2 N \times 2 N)$ pixels. Using Fourier transform, a $(2 N \times 2 N)$ spectrum image could be obtained. The latter image contains the phase spectrum information $(\phi)$. Hereinafter, a new method which could reduce the size of these phases is developed. In this case, we achieve the reduction of the needed zeros padding and the bit numbers used to code these phases.

2. Receiver (Figures 2-b and 3) is introduced to retrieve the original image (by only using half of the phase information $(\phi)$ stored and/or transmitted, see Figure (2-a)). To reconstructe the spectrum of the image (amplitude and phase: $A \exp (i \phi)$ ) our algorithm could be initialized by a uniform amplitude $(A=1)$. Then, an inverse Fourier transform (IFT) should be performed. Afterward, the module of this reconstructed image becomes an image of $(N \times N)$ pixels. Then, we add zeros padding to the reconstructed image to perform its Fourier Transform in order to retrieve a reconstructed image spectrum $A^{\prime} \exp \left(i \phi^{\prime}\right)$. The last obtained amplitude $A^{\prime}$ is presented the initial amplitude at the second iteration of our algorithm. The iterations should be repeated till the final convergence of our algorithm. We should mention that as the number of iterations increase, reconstructed image will become more and more similar to the original image.

At first, the target image $\left(\mathrm{I}_{0}\right)$ should be resized by adding zero columns and rows. This procedure is similar to zero-padding technique widely used in signal processing to refine the spectrum. In fact, adding zero in time domain is equivalent to adapt the sampling frequency (i.e. oversampling technique) in frequency domain. By doing so, more details can be obtained from the spectrum which allows us to well classify and select important frequency. In our application, zeros should be added to double the size of the original image. Therefore, for a target image (Figure 2-c) with $(N \times N)$ pixels, we should add $3 \mathrm{~N}^{2}$ zeros to resize the image to $(2 N \times 2 N)$ pixels (Figure 2-d). It is well known that the FT of a real image has symmetrical properties [3]. By relying on these properties, half of the spectrum can be neglected (Figure 2-a). The remaining phase information required $Q$ bits to be quantized:

$$
Q=\frac{(2 N \times 2 N) \times 2^{d}}{2}=2 N^{2} \times 2^{d},
$$

Where $d$ is the number of bits used in the quantization of the spectral phase information. 


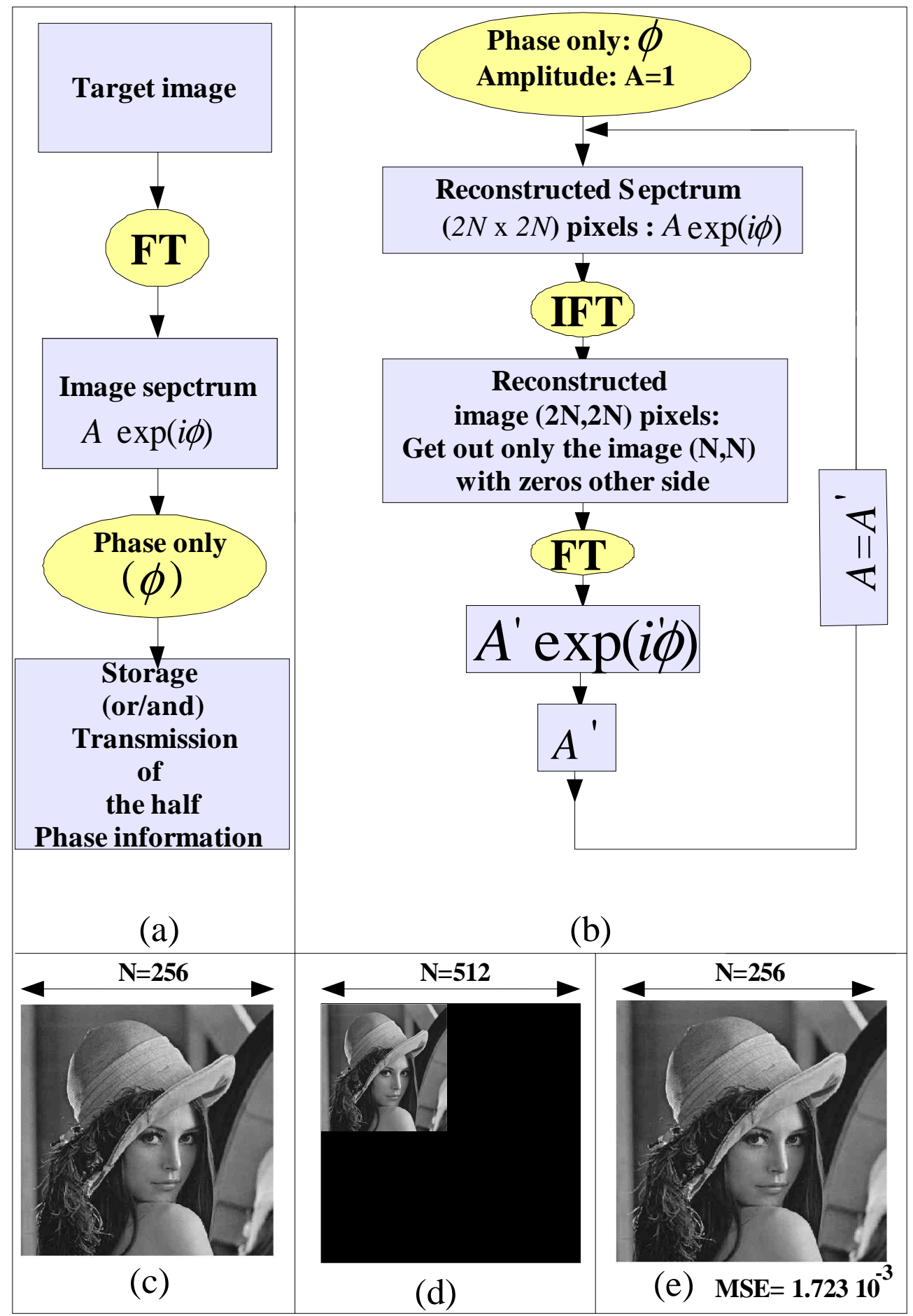

Figure 2. Main algorithm (a) transmitter, (b) receiver, (c) target Image $(N \times N)$, (d) target Image with zeros padding $(2 N \times 2 N)$, (e) output reconstructed image with 500 iterations. 
New spectral image compression method based on an optimal phase coding and the RMS duration principle
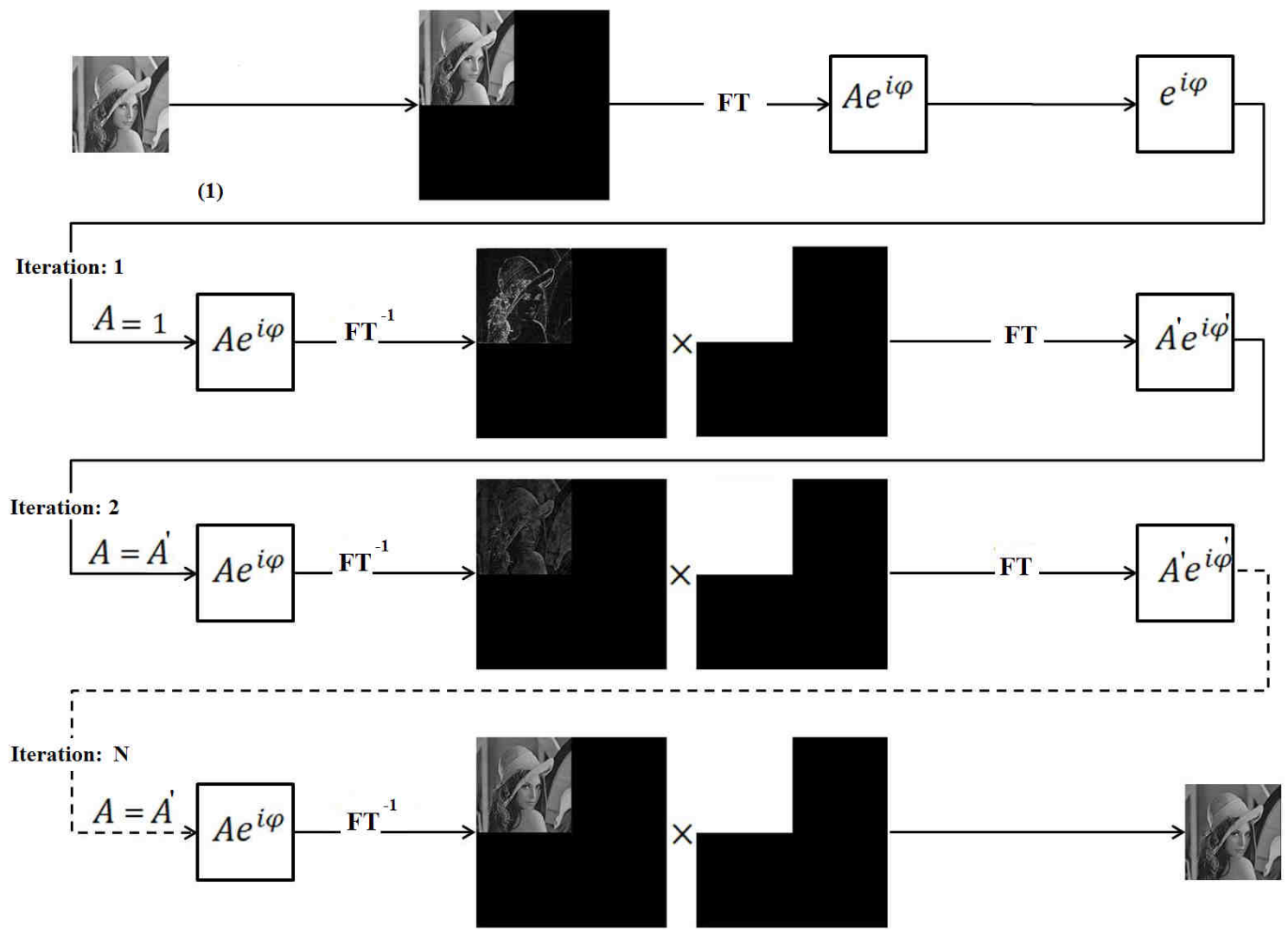

Figure 3. Image reconstructed with an iterative algorithm.

\subsection{Convergence of our reconstructed image algorithm}

Our goal, i.e. the image reconstruction by only using half of the phase information $(\phi)$, can be realized by performing a number of successive Fourier transforms, and then the final output image asymptotically tends to the target image « $I_{0} »$ Figure $(2-\mathrm{c})$. The iterative procedure should be initialized by the original phase (which is the spectral phase of the target image). At every iteration, the spectrum amplitude $(A)$ should be changed by the spectrum amplitude of the reconstructed image $A^{\prime}$. This new spectrum amplitude can be obtained by only considering the reconstructed target image $(N \times N)$ pixels (at the output plane) and by putting zeros elsewhere to get an image of $(2 N \times 2 N)$ pixels.

Then, a Fourier transform should be performed on the $2 \mathrm{Nx} 2 \mathrm{~N}$ image to obtain $A^{\prime}$. Our algorithm should be stopped when the final obtained image « $I_{n} », n$ stands for the iteration number, becomes very similar to the target image $« I_{0} »$. To test the similarity i.e. the convergence of our recontracted algorithm, a Mean Square Error (MSE) criterion is used: 
New spectral image compression method based on an optimal phase coding and the RMS duration principle

$$
M S E_{n}=\frac{1}{N^{*} N} \square_{i}^{N} \square_{j}^{N}\left|I_{0}(i, j)-I_{n}(i, j)\right|^{2}
$$

Good performance results have been observed in our conducted simulations. Figure (4) shows the reconstructed images obtained using 1, 10, 50 or 500 iterations. After 500 iterations, a MSE equal to $M S E_{500}=1.723 \cdot 10^{-3}$ has been evaluated. The latest value is 123 times less than the one obtained at the first iteration, $M S E_{1}=0.21$.

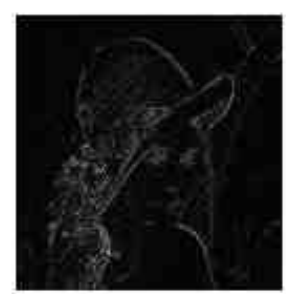

Oneiteration

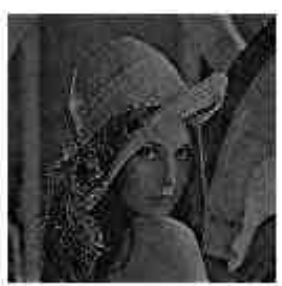

T'en iterations

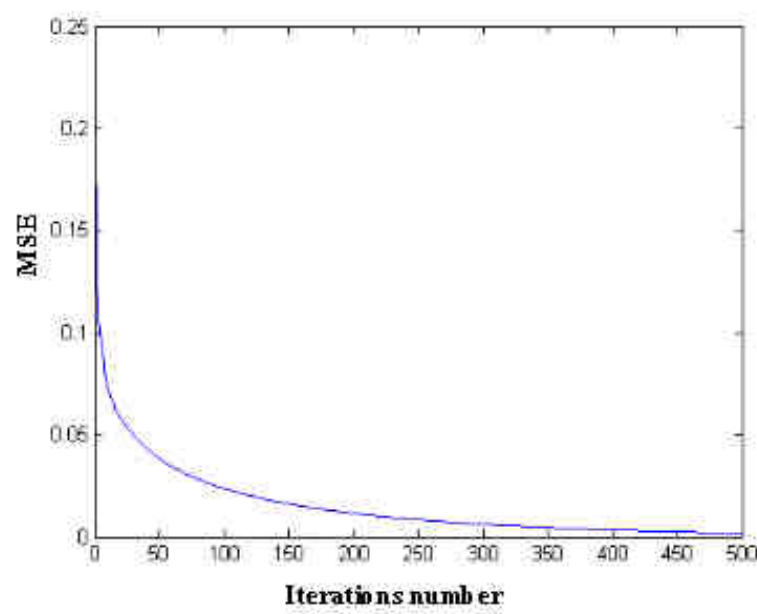

Iterations numb er

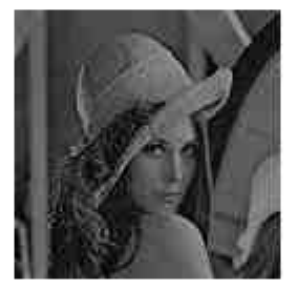

Fif ty iter ations

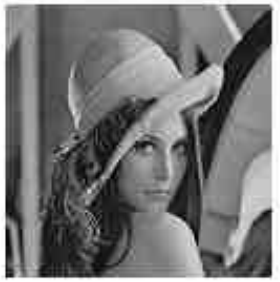

Five humdred iterations

Figure 4. Algorithm convergence.

\subsection{Influence of the number of iterations on the reconstructed image quality}

Table 1 shows the influence of the number of iterations on the quality of reconstructed images. Indeed as shown in this table, we can observe that less than 100 iterations are enough to get very good performances. First column presents the iteration number. The target image is presented in the $2^{\text {nd }}$ column. Reconstructed images, using the algorithm previously detailed, are shown in the 3rd column. The ratio given in the last column of table (1) is defined by Ratio $_{i}=\frac{M S E_{i}}{M S E_{1}}$, where $\mathrm{MSE}_{\mathrm{i}}$ is the mean square error at the $i^{\text {th }}$ iteration. For multimedia and video applications, the number of needed iterations can be reduced by introducing estimated amplitude of a reference image. Our simulations results show that a convergence can be always reached, besides that good reconstructed images could be obtained after 50 iterations. For this reason, a hundred has been fixed as the maximum number of iterations.

\subsection{File size of the needed phase information.}

Our simulation results show that, a good convergence can be achieved and the half of the spectral phase information is enough to reconstruct the whole target image. By considering half of the phase, we could compress the required information. However, the used zero-padding technique increases the 
New spectral image compression method based on an optimal phase coding and the RMS duration principle

size of the processed image. To clarify this idea, let us consider a $(256 \times 256)$ input image quantized using 8 bits. The size of such image should be $(256 \times 256) \times 8 \approx 524$ Kbit. To achieve a fast convergence speed, the zero-padding step doubles the size of the image. Therefore the obtained spectrum becomes $(512 \times 512)$ pixles. Since the reconstruction is carrying out using half of the spectrum and as Matlab performs FFT using 64 bits, the size of the final spectrum becomes $\frac{(512 \times 512)}{2} \times 64 \approx 8388 \mathrm{kbit}$ (spectrum phase). The final size is much larger than the original one.

Table 1.The number of iterations versus the quality of output reconstructed images.

\begin{tabular}{|c|c|c|c|c|}
\hline $\begin{array}{l}\text { Iterations } \\
\text { number }\end{array}$ & $\begin{array}{c}\text { Target } \\
\text { image }\end{array}$ & $\begin{array}{c}\text { Reconstructed } \\
\text { Image }\end{array}$ & MSE & Ratio \\
\hline 1 & & & $211.35 \mathrm{co}^{-3}$ & 1 \\
\hline 5 & & & $95.071^{-3}$ & 2.2 \\
\hline 10 & & & $74.521^{-3}$ & 3 \\
\hline 25 & & & $53.711^{-3}$ & 4 \\
\hline 50 & & & $38.311^{-3}$ & 5,5 \\
\hline 100 & & & $23.7510^{-3}$ & 9 \\
\hline 250 & & & $8.3210^{-3}$ & 26 \\
\hline 500 & & & $1.7210^{-3}$ & 123 \\
\hline
\end{tabular}


New spectral image compression method based on an optimal phase coding and the RMS duration principle

It is clear that in order to compress the file size, one should firstly reduce the size of the images generated with zero-padding. At the same time, we could also reduce the number of bits « $\mathrm{m} », \mathrm{~m}$ $<<64$, used to code the spectrum phase information. This procedure is further discussed in sections 3 and 4.

\section{Compression approach}

The main drawback of the approach, detailed in section 2, is still the file size of the phase information needed to reconstruct a target image. As it was previously pointed, the zero-padding increase the size of our images and their spectra. In order to solve this problem, two options have been considered:

1. First of all the effect of the number of added zeros in the zero-padding operation should be investigated in order to optimize this one.

2. For all frequencies, i.e. phase information, the number of quantization bits was also optimized.

\subsection{Optimizing the zero-padding.}

Here, the relationship between the frequency oversampling factor and the quality of reconstructed image is investigated. Let us consider a gray scale image shown in Figure 5 vith $(256 \times 256)$ pixels (each pixel is coded on 8 bits) as the input of our iterative algorithm illustrated in Figure 2.

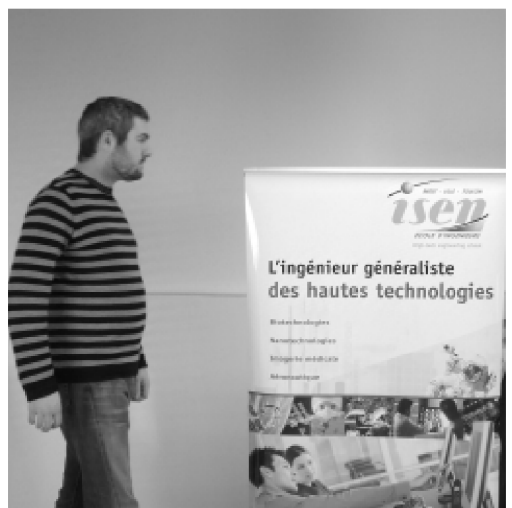

Figure 5. Florent's image, $(256 \times 256)$ pixels, 256 gray levels ( 8 bits $)$.

To conduct our simulations, half of the spectrum plane is selected, constant amplitude equal to one is considered (as an initialization of our algorithm), Fourier phase is evaluated in $[-\pi, \pi]$ and 8 bits are used in the quantization part. During the simulations, an increased number of zeros has been introduced in the zero-padding step. According the simulation presented in table 2, we can notice the following:

- Compression ratio, defined by equation (3) as the size ratio of original and reconstructed image, decreases with an increasing number of zeros included in the zero-padding:

$$
C_{r}=\exists-\frac{\text { final size }}{\text { original size }} \boxminus \times 100
$$

- The quality of reconstructed image increases if the number of zeros is increased.

- A fast convergence was observed. 
New spectral image compression method based on an optimal phase coding and the RMS duration principle

- With only $30 \%$ of added zeros, satisfactory results have been obtained, see table 2 .

- An overall compression is noticed when the added zeros are less than $45 \%$ of the original size of the image size: The sign "-" means that the resulting file size to be stored and/or transmitted is bigger than the original one.

- If the added zeros are less than $15 \%$, we notice that the convergence was not always guaranteed.

Table 2. Effect of the number of zeros padding on the quality of reconstructed images

\begin{tabular}{|c|c|c|}
\hline $\begin{array}{l}\text { Zero padding } \\
\text { expressed in } \\
\text { percentages }\end{array}$ & $\begin{array}{l}\text { Reconstructed } \\
\text { image }\end{array}$ & $\begin{array}{l}\text { Normalized } \\
\text { MSE }\end{array}$ \\
\hline $\begin{array}{c}100 \% \\
(256,256) \\
\overrightarrow{(512,512)}\end{array}$ & $\mathrm{Cr}=-100 \%$ & 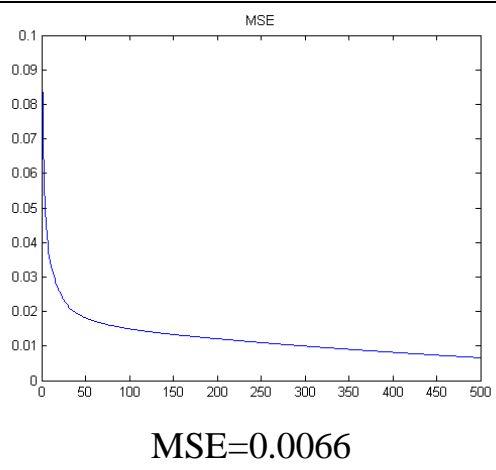 \\
\hline $\begin{array}{c}60 \% \\
(256,256) \\
\rightarrow \\
(409,409)\end{array}$ & $\mathrm{Cr}=-27 \%$ & 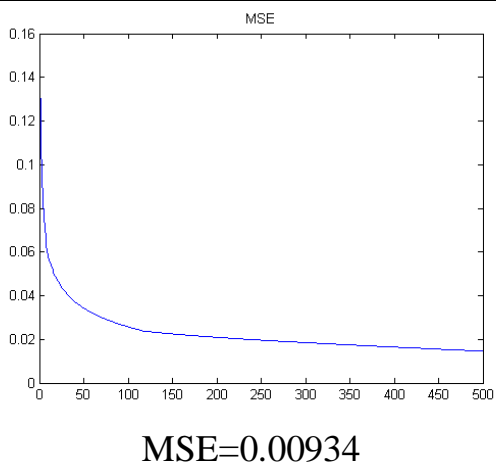 \\
\hline $\begin{array}{c}40 \% \\
(256,256) \\
\rightarrow \\
(358,358)\end{array}$ & $\frac{}{\mathrm{C}=+2.2 \%}$ & 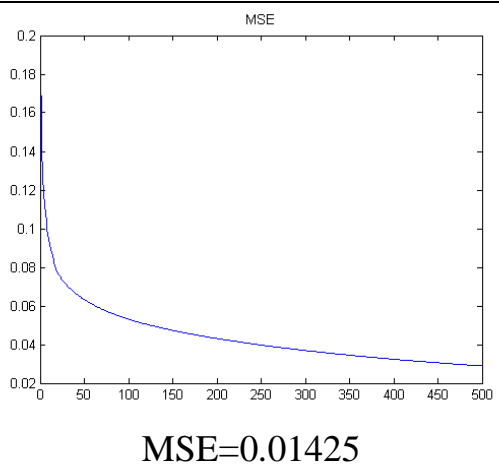 \\
\hline
\end{tabular}


New spectral image compression method based on an optimal phase coding and the RMS duration principle

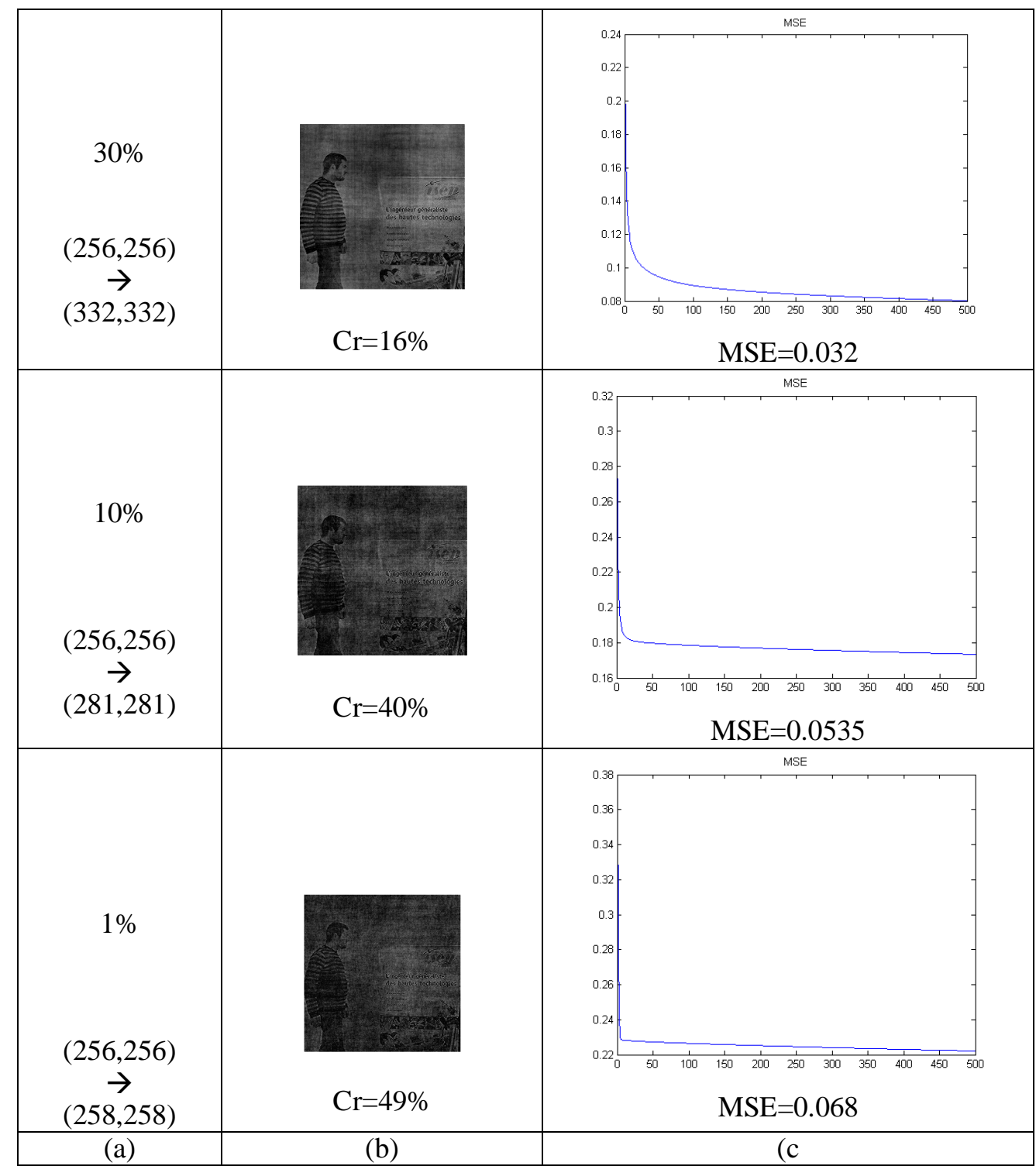

As the convergence can't be guaranteed below $15 \%$ of added zeros and compression effect will be lost if we reach $45 \%$, therefore we consider in our simulations $\mathbf{3 0 \%}$ of added zeros, see table 2 . In this case, a compression ratio of $16 \%$ is observed. To further enhance the compression ability of our algorithm, an optimization of quantization bit number is conducted; more details are given in the following section.

\subsection{Bit reduction}

In this section, we test the influence of the quantization bit number on the performance of our algorithm. At first, we should mention that Matlab uses 64 bits to code the spectrum. As we are only interested in the phase information which are bounded by $[-\pi, \pi] ; 64$ bits are extremely high number. In order to solve that problem, we adapted the coding technique proposed by DRAKIS et al in [18-19]. 
New spectral image compression method based on an optimal phase coding and the RMS duration principle

By reducing the used bit number from 64 to 8 , we could not notice a big difference, see table 3 (to perform these simulations, $30 \%$ of zeros have been added to the original image, which is a $(256 \times 256)$ pixels, see table $(2)$. The new compression rate is expressed as following:

$$
C_{r}=\square-\frac{\text { final size }}{\text { original size }} \square \times 100=\square-\frac{(N+m) \times(N+m) \times N b}{N \times N \times 8} \square \times 100
$$

where $(N \times N)$ stands for the size of original image (without zeros padding), $m$ is the number of added zeros in the zeros-padding stage and the number of used bits is denoted by $\mathrm{Nb}$.

Table 3. Effect of the bit number on the quality of reconstructed image

\begin{tabular}{|c|c|c|c|}
\hline $\begin{array}{c}\text { Bit } \\
\text { Numbe } \\
r\end{array}$ & $\begin{array}{l}\text { Compression } \\
\text { ratio }\end{array}$ & $\begin{array}{l}\text { Reconstructed } \\
\text { image }\end{array}$ & thents \\
\hline 8 & $16 \%$ & ${ }_{m \rightarrow 1}$ & 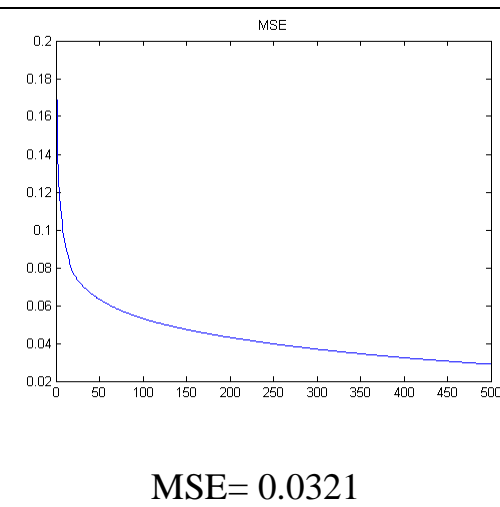 \\
\hline 7 & $26 \%$ & 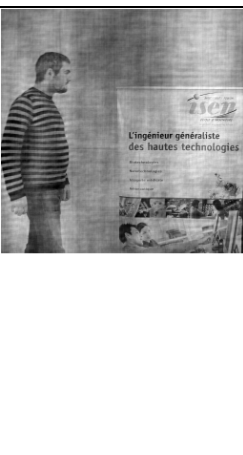 & MSE $=0.0351$ \\
\hline
\end{tabular}


New spectral image compression method based on an optimal phase coding and the RMS duration principle

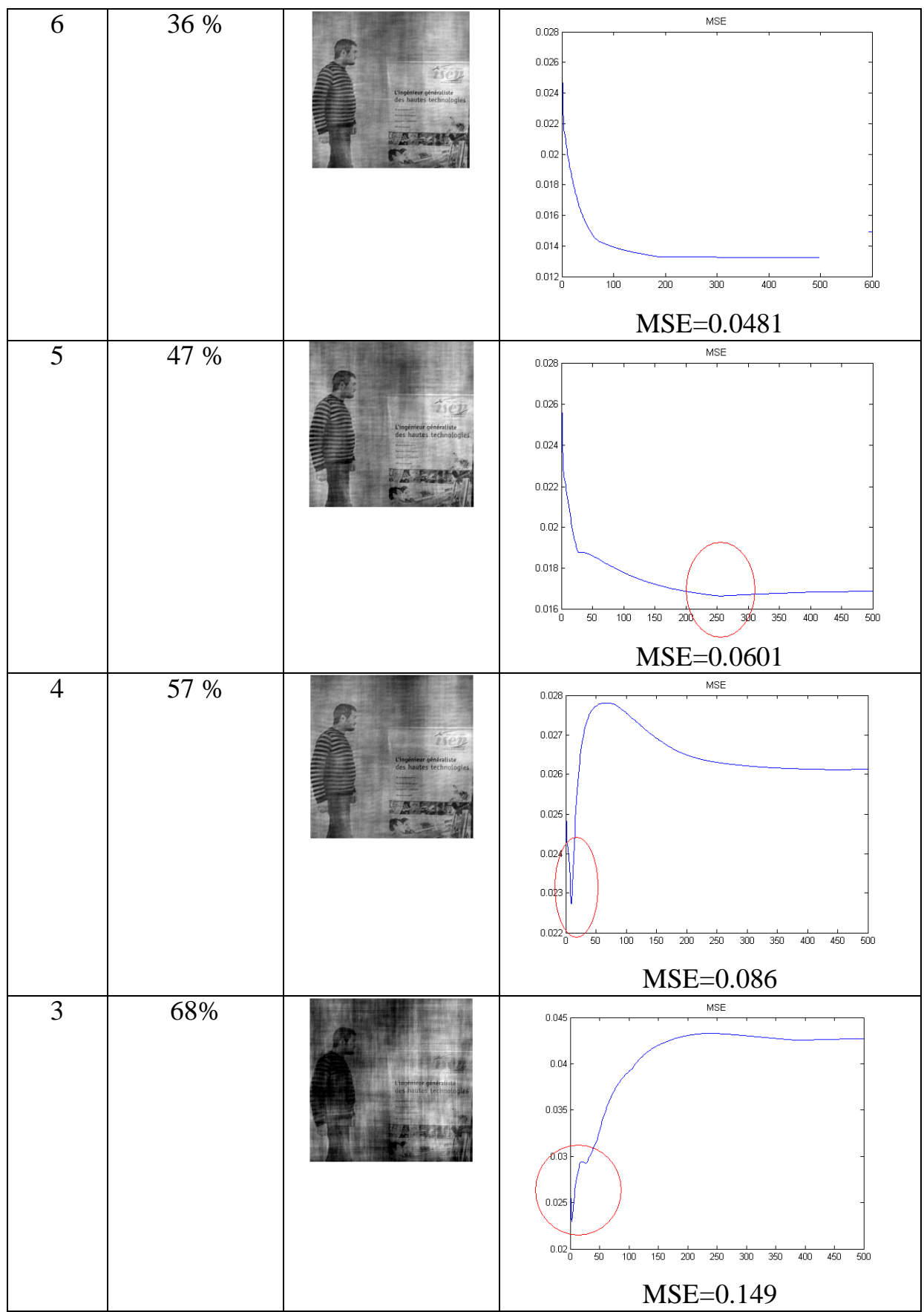


New spectral image compression method based on an optimal phase coding and the RMS duration principle

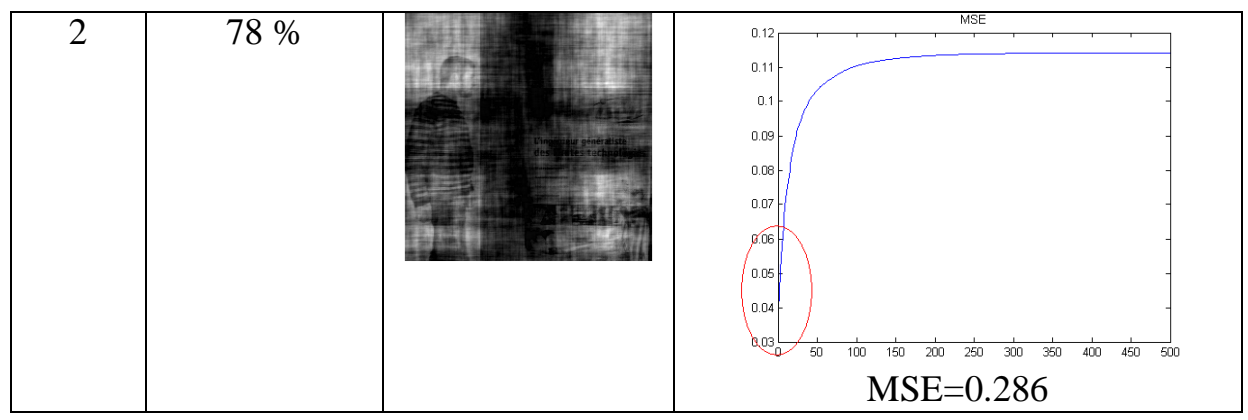

Table 3 clearly shows that 8 bits are enough to achieve good results. If less than 8 bits are used then the image quality is deteriorated. Besides that a bit number, lower than 4 , could affect seriously the convergence of the algorithm. Therefore, we should select the number of added zeros and the number of bits to reach a compromise among the quality of the obtained images, the compression ratio and the algorithm convergence. A simple compromise could reach by selecting a $30 \%$ of zero-padding $(\mathrm{m}=$ 76) for a $(256 \times 256)$ images and the bit number could set constant. However, a better compression rate could be achieved if the bit number has been selected accordingly to the importance of the frequency in the spectrum plane. This new technique is described better in the following section.

\section{Bit number based on the RMS duration criterion}

In previous sections, we showed that the proposed algorithm can give good results however we should reach a compromise between quality and compression ratio. Besides that, we discussed the impact of the number of zero added during the zero-padding step and the number of bit allocated in the quantization or the spectrum calculus.

In this section, we focus on the fact that a variable bit number could achieve better performance. The number of bits, used to code the phase information, is selected between 2 and 8 bits and depends on the local importance of the phase information Figure 6. Highly important phase information should receive a large bit number (8). The importance of the phase information is determined by Papoulis's criterion called RMS-DURATION [22].

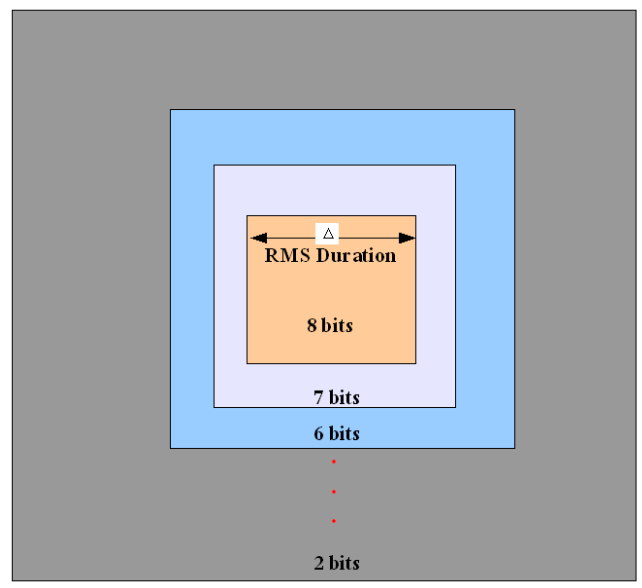

Figure 6. Bit number based on RMS_Duration criterion applied in Fourier's domain. 
To simplify our discussion and clarify our ideas, let us briefly describe Papoulis's criterion. RMSDuration (useful bandwidth) is a spectral criterion which can split the spectrum plane into various area of similar level of energies. At first, we should consider the extent of a two dimensional signal $\mathrm{I}(\mathrm{x}, \mathrm{y})$, which spectrum is $S_{I}(u, v)=A(u, v) \times \exp \{i \psi(u, v)\}$. To define the extent " $\Delta$ " (Figure 6), we opt in this work the RMS signal duration estimated according to an approximation formula proposed in [22]. The RMS duration is often approximated by a 2D intergarl of the second moment of the signal as

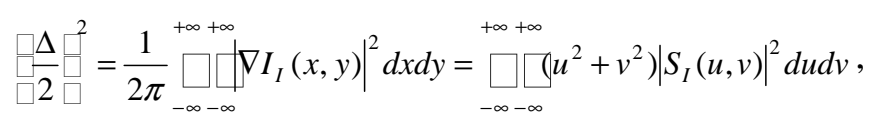

where $\nabla I(x, y)$ is the gradient of the image and $\frac{1}{2 \pi}$ is an angular normalization factor.

Simulation results, see Figure 7, prove the efficiently of such optimization. Indeed a good compression ratio, $\mathrm{C}_{\mathrm{r}}=60 \%$, and good quality of reconstructed image have been observed. Indeed, Figures (6-a) and (6-b) show a very good convergence with a low value of MSE. We should mention here that by adjusting the size of the RMS-Duration, we could improve the compression ratio.

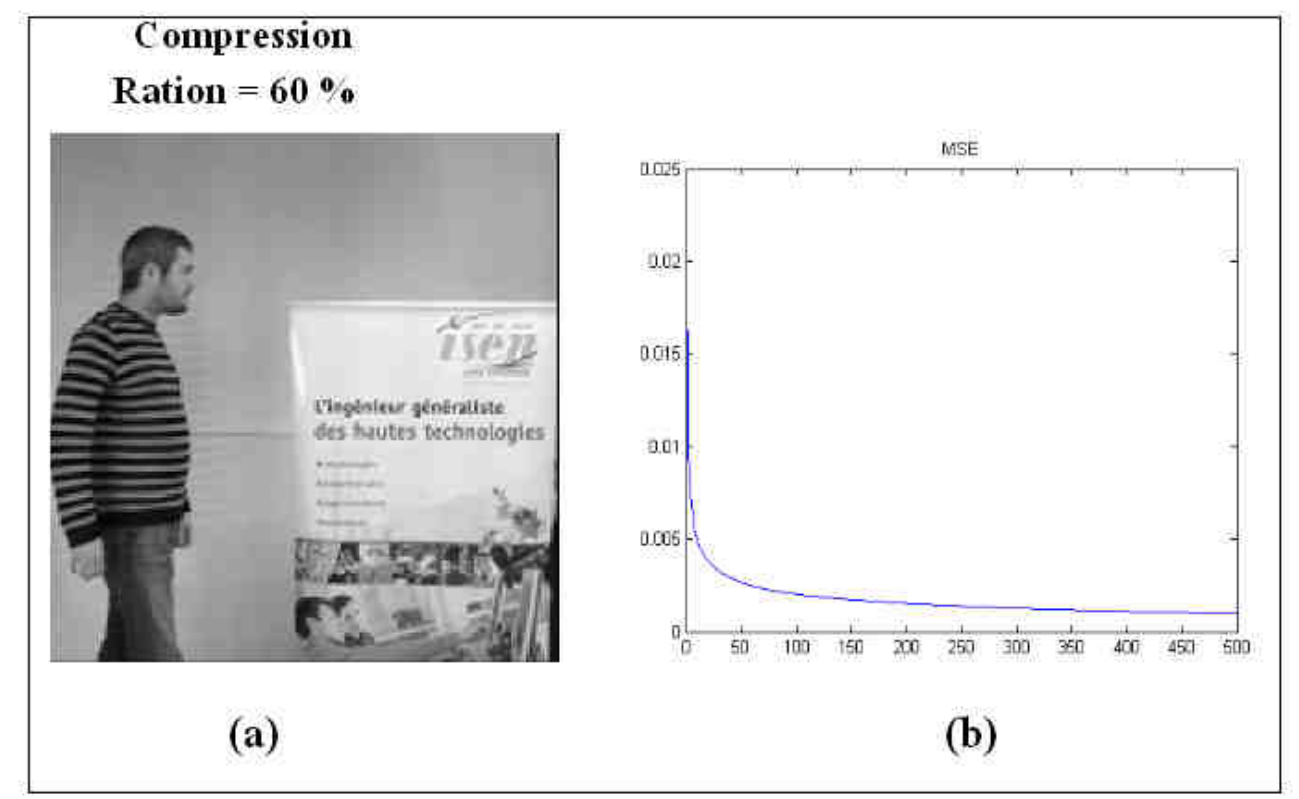

Figure. 7. Simulations results obtained with optimization of the used bit number.

\section{Conclusions}

In many applications such as face recognition, security check, etc, an identification of target image is still the main task to perform. In most this recent security applications, video sequences (containing target images) should stored or transmitted to be remotely processed in headquarters. In those applications, transmitting compressed and pre-processed images becomes their major priority. In this 
New spectral image compression method based on an optimal phase coding and the RMS duration principle

case, our new proposed approach could be of great interest. In fact, using our approach, we could easily compress a target image. The new proposed approach is only based on the use of the Fourier phase information. The main idea consists on applying an iterative algorithm on the phase information. It has been shown that number of added zero in the zero-padding step and the quantization bit number could be optimized to reach a compromise between the size of the final image and the quality of such images. A compression ratio of $60 \%$ was obtained. Moreover, various simulations showed that our reconstructed image doesn't affect the correlation decision.

\section{References}

[1] Alfalou A. and Brosseau C. 2009 Optical image compression and encryption methods Adv. Opt. Photon. 1 589-636.

[2] Alfalou A. and Brosseau C. 2010 Exploiting root-mean-square time-frequency structure for multiple-image optical compression and encryption Opt. Lett. 35 1914-16.

[3] Goodman J. W. 1968 Introduction to Fourier Optics McGraw-Hill.

[4] VanderLugt A. B. 1964 Signal detection by complex spatial filtering IEEE Trans. Inf. Theory IT$10139-45$.

[5] Horner J. L. and Gianino P. D. 1984 Phase-only matched filtering Appl. Opt. 23 812-16.

[6] B. Javidi, S. F. Odeh, and Y. F. Chen, "Rotation and scale sensitivities of the binary phase-only filter." Appl. Opt. 65, 233-238.

[7] Alfalou A., Keryer G. and de Bougrenet de la Tocnaye J. L. 1999 Optical implementation of segmented composite filtering Appl. Opt. 38 6129-36.

[8] Alfalou A. and Brosseau C 2010 Understanding Correlation Techniques for Face Recognition: From Basics to Applications in Face Recognition, In-Tech, ISBN 978-953-307-060-5.

[9] Refregier P. and Javidi B. 1995 Optical image encryption based on input plane and Fourier plane random encoding Opt. Lett. 20 767-69.

[10] Unnikrishnan G. Joseph J. and Singh K. 2000 Optical encryption. by double-random phase encoding in the fractional Fourier domain Opt. Lett. 25, 887-89.

[11] Kishk S. and Javidi B. 2002 Information hiding technique with double phase encoding Appl. Opt. 41 5462-70.

[12] Alfalou A., Mansour A., Elbouz M. and Brosseau C. 2010 Optical compression scheme to multiplex \& simultaneously encode images In Optical and Digital Image Processing Fundamentals and Applications (to be published).

[13] Alfalou A.and Mansour A. 2009 A new double random phase encryption scheme to multiplex \& simultaneous encode multiple images Appl. Opt. 48 5933-47.

[14] Naughton T.J., Frauel Y., Javidi B., and Tajahuerce E., 2002 Compression of digital holograms for three-dimensional object reconstruction and recognition Appl. Opt. 41 4124-31.

[15] Oppenheim A.V. Lim J.S. 1981 The importance of phase in signals Porc. Of the IEEE $69529-$ 41.

[16] Quatieri T.Jr., Oppenheim A.V.1981 Iterative techniques for minimum phase signal reconstruction from phase or magnitude IEEE Transactions on Acoustics, Speech and Signal Processing, ASSP-29 1187-93.

[17] Huang T.T., Sanz J.L.C., Blanz W.-E. 1988 Image representation by one-bit Fourier phase: theory, sampling,and coherent image model IEEE Transactions on Acoustics, Speech and Signal Processing, 36 1292-1304.

[18] Darakis E. and Soraghan J. J. 2006 Compression of interference patterns with application to phase-shifting digital holography Appl. Opt. 45, 2437-43.

[19] Darakis E. and Soraghan J.J. 2007 Reconstruction domain compression of phase-shifting digital holograms Appl. Opt. 46 351-56. 
New spectral image compression method based on an optimal phase coding and the RMS duration principle

[20] Alfalou A., Elbouz M., Jridi M., Loussert A. 2009 SPIE Proceeding Vol. 7486, Optics and Photonics for Counterterrorism and Crime Figurehting V, Colin Lewis Editors, SPIE Europe Security+Defence, Berlin, 74860J.

[21] Behar J., Porat M., Zeevi Y.Y. 1992 Image reconstruction from localized phase Transactions on Signal Processing, IEEE 40 736-43.

[22] Papoulis A. 1962 The Fourier Integral and its Applications McGrawHill, New York.

[23] ElBouz M. and Heggarty K. 2000 Signal window minimum average error algorithm for multiphase level computer-generated holograms Optics Communications 180 21-28. 